\title{
La alternativa de Hedley Bull frente a las propuestas realistas y liberales como marco para el análisis de las relaciones internacionales en Latinoamérica*
}

\author{
Hedley Bull's Alternative in Contrast to Realist \\ and Liberal Proposals as a Framework for the \\ Analysis of International Relations in Latin \\ America
}

Juan Francisco Gómez Montoya ${ }^{* *}$

Recibido: 13 de mayo 2015

Aprobado: 8 de julio de 2015

Disponible en línea: 30 de junio de 2016

\section{Resumen}

El presente artículo busca presentar la alternativa teórica de Hedley Bull y la Escuela Inglesa de las Relaciones Internacionales, ante los grandes paradigmas del estudio de las relaciones internacionales, alternativa que tiene la aceptación de varios académicos tanto dentro del Reino Unido como en otros países. Actualmente, existen en Latinoamérica discursos académicos y políticos muy fuertes basados en las dos grandes escuelas de las Relaciones Internacionales, a saber: realismo y liberalismo. El presente artículo analiza ambas escuelas, el debate que actualmente tiene lugar entre ellas, su materialización en los discursos más utilizados dentro del continente y

\section{Abstract}

This article purports to present Hedley Bull and the English School of International Relations's theoretical alternative in contrast to great paradigms for the study of international relations, having such alternative the acceptance of several scholars both in the UK and other countries. Currently, there are very strong speeches within Latina America based on the great schools of International Relations, namely realism and liberalism. This article analyzes both schools, the current debate among them, its materialization into the most common speeches within the continent and presents the proposal formulated by Hedley Bull and the English School of

doi:10.11144/Javeriana.papo21-1.ahbp

* Artículo de revisión.

** Politólogo por la Pontificia Universidad Javeriana con énfasis en Gestión Pública, magíster en Estrategia y Geopolítica por la Escuela Superior de Guerra Teniente General Luis María Campos (Buenos Aires), magíster en Relaciones Internacionales Económicas y Políticas Europa-América Latina por la Universitá di Bologna (Italia), especialista en Defensa Nacional por la Escuela Superior de Guerra de la Universidad Militar Nueva Granada y especialista en Gestión Periodística por la Universidad de Buenos Aires. Correo electrónico: dynghedu@hotmail.com 
da a conocer la propuesta planteada por Hedley Bull y la Escuela Inglesa de las Relaciones Internacionales como una opción adicional para analizar y entender la realidad del continente.

\section{Palabras clave}

realismo; liberalismo; Hedley Bull; Escuela Inglesa de las Relaciones Internacionales

\section{Cómo citar este artículo:}

Gómez Montoya, J. F. (2016). La alternativa de Hedley Bull frente a las propuestas realistas y liberales como marco para el análisis de las relaciones internacionales en Latinoamérica. Papel Político, 21(1), 225-248. http://dx.doi. org/10.11144/Javeriana.papo21-1.ahbp
International Relations as an additional option to understand the continent's reality.

\section{Keywords}

realism; liberalism; Hedley Bull; English School of International Relations 
"La lucha por el poder es universal en tiempo y espacio y es un hecho innegable de la experiencia”, decía Morgenthau (1978, p. 29), el conocido padre de la Escuela Realista de las Relaciones Internacionales. Con este racionamiento, la Escuela Realista se posicionó como la escuela más fuerte del estudio de las relaciones internacionales, hasta que el liberalismo propuso algunas alternativas que se alejaban de la constante lucha por el poder entre las naciones, que inició un largo debate académico entre exponentes de una y otra corriente. Los realistas asumen que en la política internacional existen relaciones verticales de poder donde los más débiles se rinden ante los designios de los más fuertes. El liberalismo, en cambio, asume que las relaciones internacionales están signadas por relaciones horizontales de poder, en las que las naciones libres interactúan entre sí, siempre pensando en su propio bienestar.

Por otro lado, si se considera como un hecho que toda acción y discurso político tiene un trasfondo teórico, aunque este no sea conocido para el emisor del discurso, entonces claramente ambas corrientes han sido los principales soportes de los grandes discursos que se escuchan en el continente. El realismo, la corriente más fuerte, es un gran soporte para muchos discursos políticos de la región, aunque probablemente esto sea ignorado por muchos de sus oradores. Por ejemplo, cuando el expresidente Hugo Chávez (2013) vociferaba: "La confrontación aquí no es del majunche contra Chávez, es de la burguesía contra el pueblo, es del imperio contra la Patria”, es un discurso que claramente está soportado sobre una visión realista de las relaciones internacionales, del mismo modo como lo es el discurso de Mujica (2013) cuando afirma: "Es posible un Mundo con una humanidad mejor, pero tal vez hoy la primera tarea sea salvar la vida". Del mismo modo, el continente ha conocido presidentes de corte netamente liberal, como es el caso de Óscar Arias (2009), expresidente de Costa Rica, cuando se pregunta:

¿Qué hicimos mal? No puedo enumerar todas las cosas que hemos hecho mal. Para comenzar, tenemos una escolaridad de 7 años. Esa es la escolaridad promedio de América Latina y no es el caso de la mayoría de los países asiáticos. Ciertamente no es el caso de países como Estados Unidos y Canadá, con la mejor educación del mundo, similar a la de los europeos. De cada 10 estudiantes que ingresan a la secundaria en América Latina, en algunos países solo uno termina esa secundaria. Hay países que tienen una mortalidad infantil de 50 niños por cada mil, cuando el promedio en los países asiáticos más avanzados es de 8, 9 ó 10.

Es importante hacer una anotación, ya que seguramente se podrá argüir que el soporte teórico de varios de los presidentes de Latinoamérica es el marxismo o su variante el neomarxismo en lugar del realismo. Sin embargo, es importante mencionar que, en las relaciones internacionales, aunque existan sociólogos internacionales, como Immanuel Wallerstein de corte netamente marxista, es difícil pensar en una sólida corriente de 
relaciones internacionales netamente marxista, básicamente porque el planteamiento marxista se encontraría con el obstáculo de la anarquía en las relaciones internacionales, por el hecho de que no todos los Estados que conforman la política internacional son capitalistas, e incluso hay Estados poderosos que son más proclives a la izquierda y, además, entre otros argumentos, las relaciones internacionales no están signadas por relaciones laborales de ningún tipo, lo cual derrumba los pilares iniciales de esta corriente teórica sobre relaciones internacionales. Sin un Estado central que ejecute las reglas en favor de una clase beneficiada, sin una clase beneficiada específica (ya que claramente no existe criterio alguno para defender la tesis de que Estados tan poderosos y tan disímiles como China, los Estados Unidos y Rusia hacen parte de una misma clase) y sin relaciones laborales entre los miembros, que, al fin de cuentas, son las que dan nacimiento al proletariado, no es posible hacer un análisis marxista del sistema internacional.

Por ende, el discurso de aquellos presidentes que hablan de socialismo, revolución e imperio, es un discurso de corte realista, el cual trata las relaciones verticales de poder entre Estados.

Claramente, los exponentes de realismo y liberalismo han mostrado argumentos muy fuertes en su favor, y estas dos corrientes son las corrientes más fuertes dentro del campo, debido a que hay un gran número de estudios que las soportan, pero ċson las únicas? ¿Puede el liberalismo superar las críticas del realismo, y viceversa? ¿Cómo se explica desde el realismo el auge y el declive de una superpotencia como los Estados Unidos, desde un punto de vista exclusivamente político y militar? ¿Cómo explica una escuela liberal como el neofuncionalismo los resultados de la Asociación Latinoamericana de Libre Comercio? ¿Cómo se explica desde el institucionalismo neoliberal la invasión de los Estados Unidos a Irak a inicios del siglo XXI?

Ahora bien, es claro que en las relaciones internacionales los mayores referentes son anglosajones para entender y explicar el sistema mundial. Dentro del estudio de las relaciones internacionales, las vertientes del realismo y el idealismo liberal, según Bull (2005), tienen su más remoto origen en el pensamiento hobbesiano y el pensamiento kantiano, respectivamente. Sin embargo, el mismo Bull considera que existe otra vertiente de estudio de las relaciones internacionales, que tiene su origen en los planteamientos de Hugo Grocio, situado en medio de las dos anteriores. Dentro de las distintas escuelas que han intentado soportar sus planteamientos bajo la visión grociana de las relaciones internacionales, se encuentra la famosa Escuela Inglesa, y su mayor exponente, Hedley Bull.

Hedley Bull fue un académico australiano del siglo XX, quien curiosamente fue conocido como el "padre de la Escuela Inglesa de las Relaciones Internacionales". Sus ideas logran conciliar los planteamientos realistas y liberales, razón por la cual no es formalmente incluido como parte de alguna de las dos escuelas. Realistas como Hoffman (1986) alaban que rescate conceptos realistas, como la autoayuda y la anarquía en 
el sistema internacional, a la vez que analizan instituciones (en el sentido bulliano del concepto) que son de cuño meramente realista, como es el caso de la guerra, el balance de poder y las potencias. Sin embargo, Bull mismo ha dicho considerarse heredero de la visión grociana de las relaciones internacionales, la cual "describe la realidad internacional como una sociedad de Estados o una sociedad internacional" (2005, p. 78), en la que "la actividad internacional... que mejor ejemplifica a la sociedad internacional en su conjunto, no es ni la guerra entre Estados... sino el comercio o, de forma más general, el intercambio económico y social” (p. 78.). Dicho planteamiento se acercaría más a la corriente liberal de las relaciones internacionales. Además, el mismo Hoffman reconoce:

[Bull] no inicia su estudio, como lo hacen los realistas, mirando al Estado y su poder, un concepto sobre el cual tiene poco para decir... El cuerpo completo de trabajo de Bull toma su punto de partida en el grupo, o contexto, o "ensamble" el cual es formado por los Estados cuando interactúan (1986, p. 180).

Esta inhabilidad para catalogar a Bull dentro de una u otra corriente ha llevado a que sea clasificado como parte de la corriente constructivista, aunque claramente Bull es mucho más cercano en materia teórica a Waltz (1979) que a Wendt (1992). ${ }^{1}$

El objeto principal de este trabajo, entonces, será presentar de manera muy somera el trabajo de Bull en contraste con los aportes de algunos de sus contemporáneos, para mostrar cuáles son las fortalezas de esta propuesta (no sin dejar de ignorar que evidentemente la propuesta bulliana también tiene grandes debilidades), que puede ayudar a formar una visión distinta de la realidad continental. Por esto, de manera un poco tangencial, y como un valor agregado, se pretende hacer una pequeña exposición sobre cómo los planteamientos de Bull podrían influir en un nuevo discurso político en Latinoamérica, donde la realidad no esté signada por las decisiones de un "imperio" ni por las fórmulas del Fondo Monetario Internacional o del libre comercio, sino por normas e instituciones entendidas dentro del marco conceptual bulliano, como se explicará más adelante. Siguiendo este orden de ideas, primero, se hará un breve repaso del planteamiento realista, que explica sus preceptos centrales y principales exponentes. Luego, se hará lo mismo con el planteamiento liberal, para finalmente confrontarlo con la propuesta de Bull. Al final, se esbozarán algunas conclusiones globales.

\footnotetext{
${ }^{1}$ Alexander Wendt, considerado el padre de la corriente constructivista de las relaciones internacionales, basa su trabajo en el estudio de las relaciones internacionales desde la percepción de los Estados mismos y sus dirigentes. No es este un tema único de Bull, lo mismo sucede con Barry Buzan, considerado, junto con Ole Wæver, como el padre de la escuela danesa de las relaciones internacionales. Sus contribuciones sobre el concepto de complejos de seguridad, sus análisis del concepto de la sociedad internacional y sobre el realismo estructural waltziano lo han llevado a oscilar entre realismo y constructivismo. El realismo periférico de Carlos Escudé también podría ser considerado de esta tendencia.
} 


\section{El realismo y el mundo como una lucha por el poder}

Como bien lo menciona Mearsheimer (1994), “el realismo pinta una imagen más bien desalentadora de la política mundial” (p. 9). El realismo es la primera escuela de las relaciones internacionales y una de las principales bases para analistas y teóricos. Sus concepciones fundamentales, la autoayuda (Kenneth, 1979), la anarquía en la realidad internacional (Morgenthau, 1978), el protagonismo de los Estados dentro de la política internacional (Grieco, 1988), el potencial inherente que tiene cada Estado de infligir daño a los demás, la incertidumbre que se tiene sobre las agendas e intenciones verdaderas de cada uno de los actores dentro del sistema internacional y sus constantes esfuerzos por mantener su posición estratégica en el ámbito internacional (Mearsheimer, 1994) son ampliamente compartidas por seguidores y debatidas por detractores por igual. Sin embargo, incluso acérrimos defensores de la Escuela Liberal como Keohane y Nye (1977) o Axelrod (2006) han aceptado algunos postulados del realismo y trabajado basados a ellos, como es el caso de la anarquía internacional. En síntesis, de acuerdo con los postulados realistas, cada Estado se encuentra en una eterna competencia por la obtención de poder político y militar en detrimento de los demás. Bajo esta lógica, la guerra es inevitable dentro del sistema internacional, y la probabilidad de paz y cooperación internacional se encuentra limitada por las circunstancias y siempre será un estado coyuntural.

Sin embargo, hay un axioma realista que ha repercutido en muchos intelectuales latinoamericanos y que Bull debate de manera contundente: el verticalismo de las relaciones de poder entre los Estados dentro de la política internacional. Desde el realismo clásico de autores como Morgenthau o como Carr (1946), y llegando al neorrealismo con autores como Waltz y el realismo estructural o Mearsheimer (2001) y su realismo ofensivo, los realistas han puesto marcado énfasis en el poder de las potencias y su capacidad para moldear la realidad de la política internacional a su libre albedrío.

Desde esta perspectiva, se plantea una relación vertical de poder entre el centro y la periferia, en la que la periferia se encuentra subyugada y es un actor secundario y sin importancia dentro de la política internacional.

Tanto individual como en interacción entre sí, aquellos Estados que históricamente han sido conocidos como grandes potencias y que son conocidos actualmente como las superpotencias establecen y ejecutan las reglas básicas que influyen en su propio comportamiento y el comportamiento de los Estados menos dentro del sistema (Gilpin, 1981, p. 30).

Esta concepción del sistema internacional casualmente concuerda con la de muchos autores latinoamericanos que siguen una tendencia muy difundida dentro del continente, según la cual hay una gran responsabilidad de las potencias, sobre todo las potencias 
occidentales, en la realidad del continente, de tal suerte que sus decisiones y acciones con respecto a América Latina serían los principales determinantes del destino del continente. Incluso neorrealistas como Nye (1969) defienden la idea de que los Estados Unidos es la potencia dominante del hemisferio occidental, con mayor fuerza en el Caribe, aunque lo haga desde la perspectiva de un académico estadounidense.

La concepción, entonces, de muchos pensadores latinoamericanos, no solo tiene el pensamiento realista de fondo, el cual es claramente planteado desde el punto de vista teórico por intelectuales como Escudé (1998) con su realismo periférico, sino que, además, se soporta sobre un discurso que amalgama una serie de reivindicaciones históricas de un pasado colonial, que no ha sido superado debido a los repetidos intentos de las potencias occidentales por retomar el poder y control de las vastas riquezas naturales que guarda el continente (Galeano, 2004), junto con una infinita variedad de reivindicaciones sociales, las cuales actúan como si fuesen una sola fuerza, aunque en la práctica son conflictivas entre sí. Dicha agrupación de argumentos retóricos ha sido utilizada por personajes tan disímiles como Lázaro Cárdenas, Juan Domingo Perón, José Carlos Mariátegui y Hugo Chávez.

Una revisión formal a la propuesta que hace Hedley Bull, y que es descrita más adelante, permite entender una de las grandes debilidades del realismo: su inflexibilidad para explicar el cambio en la política internacional. La concepción absoluta del poder político y militar y su excesivo énfasis en conceptos, como el balance de poder, hacen del realismo una corriente teórica perfecta para tomar una fotografía del presente, pero no permite entender el pasado y claramente lleva a entender la dinámica internacional como si fuese estática. En palabras más concretas, es claro que el realismo puede ser útil para explicar coyunturas como la situación de China frente a Taiwán, pero no logra suficiente éxito al explicar situaciones históricas, como las razones por las cuales Corea del Sur pasó de ser un país del Tercer Mundo a un país que hace parte del primero o por qué el Reino Unido perdió irremediablemente su condición de dueño del mundo en el siglo pasado.

Este desdén por los temas económicos, históricos y demás elementos del llamado soft power, es el que permite observar la importancia que tiene la seguridad para los realistas. Esta es básicamente la razón por la cual el realismo es usualmente la base teórica que utilizan analistas de seguridad internacional como Wohlforth (1999), Jenkins (2014) o Calderón (2009).

\section{La visión esperanzadora del liberalismo}

El liberalismo es una propuesta que algunos rastrean hasta el mismo Immanuel Kant, aunque sus orígenes actuales son del siglo XX. En todo caso, en sus inicios y bases, el liberalismo incluye no solo la propuesta federalista de Kant, sino también el idealismo wilsoniano e incluso lo que es conocido como el irenismo o pacifismo, aunque actualmente la propuesta liberal tiene varias corrientes menos quiméricas y 
más cercanas al realismo, como son el neofuncionalismo (Mittrany, 2001), la teoría de regímenes internacionales (Krasner, 1983), la interdependencia compleja (Keohane y Nye, 1977) y el institucionalismo neoliberal (Keohane y Martin, 1995), entre otros. Es importante hacer esta distinción, ya que la tendencia del liberalismo que se tratará en el presente artículo corresponde a este liberalismo no utópico, el cual, de hecho, tiene actualmente la mayor voz en el debate.

El liberalismo difiere del realismo en su visión más amplia de la política internacional, en la medida en que acepta otros actores dentro de la política internacional, además de los Estados, y entiende que el poder en el ámbito internacional no solo tiene una naturaleza político-militar. En palabras de Mearsheimer (1994), las relaciones internacionales se basan en "el supuesto de que la política internacional está dividida en dos reinos - seguridad y economía política- y el institucionalismo liberal se enfoca en el segundo" (pp. 15-16). Y este énfasis en las materias económicas es fundamental para el liberalismo, ya que, como se verá, la influencia de la economía en sus diferentes corrientes es bastante evidente. Además de la economía, el liberalismo también se nutre de disciplinas tan variadas, como la antropología, la sociología, el derecho y la psicología. Siguiendo esta misma lógica, el liberalismo concibe a los Estados no como actores que constantemente están buscando más poder en detrimento de sus oponentes (lo que Adam Smith consideraría como las "ventajas absolutas”), sino que son actores racionales que siempre buscarán su mayor beneficio e incurrirán en los menores costos, donde, en algunos casos, cooperarán y en otros casos competirán (lo que David Ricardo [2001] consideraría como las "ventajas relativas”).

En segunda instancia, y desprendiéndose de lo anterior, el liberalismo no concibe las relaciones internacionales desde el punto de vista de las relaciones verticales de poder, sino que tiende más a ver cierta horizontalidad en la forma como se relacionan los Estados. En otras palabras, los Estados fuertes no priman sobre los débiles, sino que todos los Estados tienen relaciones como iguales y son varios los elementos que rigen dichas relaciones. Esta divergencia de elementos hace que la escuela liberal sea mucho más compleja para análisis que el realismo. Mientras los realistas pueden acordar en varias cosas, los liberales tienen muchos menos puntos de encuentro, distintos de la polemización de los preceptos realistas, es decir, proponer que hay más actores dentro de la política internacional que los Estados, reconocer el soft power, es decir, un tipo de poder más de tipo económico o cultural además del poder político militar, debatir la relación vertical de poder entre potencias y Estados débiles, proponer la cooperación por encima de la autoayuda y, en algunos casos, incluso, sugerir que la existencia de organizaciones, reglas o regímenes internacionales son útiles para matizar la anarquía que rige dentro de la política internacional (Keohane y Martin, 1995), aunque no logran acordar cuál de estos elementos es más importante dentro del concierto internacional. 
La posibilidad del incumplimiento de una promesa puede impedir la cooperación aun cuando la cooperación puede favorecerlos a todos. Aun así, en otros momentos, los Estados alcanzan metas comunes a través de la cooperación bajo la anarquía. A pesar de la ausencia de cualquier autoridad internacional, los gobiernos frecuentemente se comprometen a líneas de acción que sean mutuamente benéficas. Y, aunque no exista ningún soberano internacional que pueda hacer cumplir los términos del acuerdo, los Estados pueden alcanzar intereses comunes a través de la cooperación tácita, la negociación multilateral y bilateral formal y la creación de regímenes internacionales (Oye, 1985, p. 1).

Visto desde corrientes como las mencionadas, el liberalismo, en primera instancia, percibe la política internacional como un espacio donde los Estados se encuentran en una especie de sociedad civil internacional ${ }^{2}$ (entendida como la suma de los actores o grupos voluntarios de actores), que restringe y determina las acciones de los Estados (Moravcik, 1992). En segunda instancia, el liberalismo tiende a favorecer ciertas fórmulas genéricas como la mejor forma de resolver situaciones o mejorar coyunturas, como es el caso del fortalecimiento comercial para el neofuncionalismo, el fortalecimiento de la democracia en el caso de la paz democrática (Doyle, 1986) o los catorce puntos de Wilson (1918) como pasos para lograr la paz mundial. Incluso organizaciones internacionales de corte netamente liberal como el Fondo Monetario Internacional, la Comisión Económica para América Latina y el Caribe o la misma Unión Europea han tendido históricamente a insistir en ciertas fórmulas o medidas necesarias para que los Estados puedan superar sus dificultades.

En América Latina, el pensamiento liberal es el que da sustento al otro discurso tan difundido en la región, es decir, el discurso tecnocrático. De acuerdo con esta lógica, la razón por la cual Latinoamérica no ha logrado entrar en el Primer Mundo no se debe a una conspiración de las potencias occidentales en su contra, sino más bien a su inhabilidad para cumplir con ciertos requisitos y medidas necesarios para alcanzar a los más avanzados.

En el caso del liberalismo, es más complejo mencionar las debilidades debido a la gran cantidad de variantes que este tiene. Grieco (1988) plantea esta crítica de manera muy precisa, aunque algo fuerte: "Finalmente, un desarrollo intelectual inconsistente puede dar lugar a explicaciones y prescripciones divergentes" (p. 2).

Del mismo modo, se podrían hacer críticas específicas a los axiomas que rigen cada una de estas variantes. El liberalismo tiene una mayor capacidad para explicar los grandes cambios en la política internacional: desde la óptica liberal es más completa la explicación del protagonismo en el ámbito internacional de un país como Corea, y de la pérdida de dicho protagonismo de un país como el Reino Unido. Ahora bien, el enfoque liberal es apropiado para explicar los grandes cambios en la política internacional, como el nacimiento de la

${ }^{2}$ Es importante diferenciar del concepto de sociedad internacional planteado por Bull, el cual se explicará más adelante. 
Unión Europea en la década de 1990, pero ninguna de las escuelas liberales tiene fortaleza para hacer análisis de coyuntura de la manera como lo hacen los realistas. En la medida en que se nutre más de la historia y la economía y menos de ciertos axiomas, ha logrado superar ciertas limitaciones que tiene el enfoque realista, pero es débil frente a otras.

En lo conceptual, y como se mencionó, algunas corrientes del liberalismo parecen hacer mucho énfasis en las instituciones y el papel que estas desempeñan dentro de la realidad internacional. Sin embargo, no parece existir consenso con respecto a la definición de dichas instituciones. Para Keohane (1984), por ejemplo, no parece existir mucha diferencia entre una institución y un régimen, para Krasner (1983), en cambio, es claro que las instituciones internacionales hacen parte de los regímenes internacionales, aunque no son sinónimos. Y con respecto a las organizaciones internacionales, para Keohane no cabe duda de que también hacen parte de las instituciones internacionales, por lo cual sería posible concebir las instituciones internacionales como una amalgama de reglas, organizaciones y prácticas en el ámbito internacional, cuya función principal es limitar el comportamiento de los Estados que actúan dentro de ellas por medio de la reducción de los costos de verificación, la creación de repetitividad y facilitando el castigo de aquellos Estados que quieran ignorar dichas instituciones.

De hecho, el mismo Oye (1985) reconoce la falta de consenso que existe en materia conceptual entre los autores más destacados en relaciones internacionales:

El problema de explicar y promover la cooperación internacional reúne muchas de las principales preguntas en las disciplinas de la economía política y los estudios de seguridad. Sin embargo, las convenciones terminológicas divergentes y aplicaciones sustantivas han impedido la comparación de respuestas (p. 2).

Adicionalmente, el liberalismo, al ampliar el rango de los actores dentro del sistema internacional, relativizó completamente el análisis al restarles importancia a los tomadores de decisiones políticas (Grieco, 1988). Para funcionalistas y neofuncionalistas, el análisis del sistema internacional debe considerar también, entre otros, las agencias internacionales especializadas en ciertos temas técnicos, los sindicatos, los partidos políticos, los gremios de comerciantes y las organizaciones internacionales (Mittrany, 1994; Haas, 1958). Los defensores de la interdependencia compleja, por el otro lado, consideran que también deben ser consideradas las corporaciones multinacionales y las coaliciones transgubernamentales (Keohane y Nye, 1977).

Por esto, la perspectiva liberal deja, en muchos casos, y parafraseando al profesor Mearsheimer, la sensación de una "falsa promesa" ante la fortaleza de sus argumentos, pero la poca correspondencia con muchos casos de la realidad, donde el realismo es más eficaz para explicar los diferentes fenómenos que se presentan. 
Sin embargo, ¿̇on estas las únicas dos opciones teóricas para servir de soporte al análisis y la resolución de problemas dentro de las relaciones internacionales para América Latina? La propuesta de Hedley Bull, aunque no es la única, podría ayudar a complementar nuestra visión sobre la realidad internacional. Es importante aclarar en todo caso que, aunque la propuesta de Bull tiene varios aspectos interesantes, no tiene un completo poder explicativo, y el intento por conciliar la propuesta liberal y realista puede, en mucho casos, terminar generando más preguntas que respuestas.

\section{Hedley Bull y el sistema internacional}

Sobre relaciones internacionales es importante mencionar que el teórico australiano Hedley Bull introdujo una noción bastante original dentro del estudio de ellas, al hacer un cambio en el enfoque epistemológico del objeto de estudio. Las críticas que inicialmente recibió el realismo con respecto a su concepción de la racionalidad de los Estados fueron uno de los aspectos que tanto liberales como neorrealistas tuvieron que reformar. Ya sea desde el concepto de estructura waltziano (Waltz, 1979) o de regímenes internacionales de Krasner (1983), existía una concepción de que, si bien las relaciones internacionales eran anárquicas, dicha anarquía gozaba de una naturaleza sistémica. Es decir, la concepción de un sistema internacional descansaba en las relaciones entre los Estados y en el impacto generado por las decisiones y acciones entre Estados. La primera diferencia que vale la pena mencionar de la concepción bulliana es que ya no trata de una suma de Estados, al no centrarse en estas categorías, sino que considera la realidad internacional como una mezcla de intereses, valores, reglas comunes e instituciones formadas por esos mismos Estados.

Por otro lado, una de las grandes diferencias entre el pensamiento realista y el pensamiento bulliano pasa por la discriminación de los Estados dentro del sistema internacional. El realismo ha tendido a creer, por la misma noción de sus postulados básicos, que son los Estados más poderosos los que determinan la esencia misma de la realidad internacional y, por ende, restan importancia a los Estados menores. Siguiendo este orden de ideas, las reglas de juego del sistema internacional están supeditadas a las relaciones de poder. Dentro de la concepción de sociedad internacional, en cambio, tal discriminación no existe. Apoyándose en el supuesto de que todas las interacciones, instituciones, normas y valores comunes que rigen a la totalidad de los miembros de una sociedad son sus determinantes, una concepción de sociedad internacional no puede simplemente entrar a estudiar a sus miembros más poderosos. El argumento en este sentido es bastante claro: si el pensamiento realista estipula que solo son los poderosos los que determinan la realidad internacional, ¿̇por qué todavía siguen sufriendo derrotas? ¿No se supone que si un actor está en capacidad de fijar las reglas de juego para los más débiles lo haría con el ánimo de nunca ser derrotado? 
Sin embargo, antes de seguir con el razonamiento, es importante hacer algunas aclaraciones conceptuales sobre la propuesta de Bull.

En principio, la gran obsesión de Hedley Bull es el orden internacional, ante el cual intenta responder tres cuestionamientos fundamentales:

1. ¿Qué quiere decir orden en el ámbito internacional?

2. ¿Cómo se mantiene el orden dentro del actual sistema de Estados soberanos?

3. ¿Es el sistema de Estados soberanos todavía un camino viable para el (según el autor) orden internacional?

La respuesta a las dos primeras preguntas básicamente conforma la columna vertebral del presente argumento; la tercera no tiene mayor relevancia para los objetivos propuestos.

En respuesta a la primera pregunta, la definición de orden que Bull propone tiene un cierto grado de complejidad, principalmente por el hecho de que el mismo Bull reconoce que el orden es solo un aspecto de la política mundial y que, de hecho, es posible un escenario de política mundial sin orden. Por eso, una primera acepción del orden hace referencia a su existencia en contraposición con el desorden. En segundo lugar, el orden se encuentra definido como "una situación o estado de las cosas real y posible y no como un valor, un fin o un objetivo" (Bull, 2005, p. 48). En este punto, es importante hacer algunas reflexiones. En primer lugar, Bull aclara que la importancia del orden no radica en su deseabilidad, sino en su valor como objeto de estudio. Consecuentemente, Bull no encuentra relación alguna entre orden y justicia, sino que el orden puede ser injusto e incluso: "Creo que en la vida social puede haber orden aun en ausencia de normas" (p. 59), aunque considera la obtención de justicia hacia los Estados menos privilegiados como una condición necesaria para alcanzar el orden mundial.

De acuerdo con Bull, el orden, en su sentido más abstracto, hace referencia al orden social entre individuos, el cual "no consiste en una pauta o regularidad en las relaciones entre individuos o grupos sino en una pauta que conduce a un resultado determinado" (p. 56).

Consecuentemente, a pesar de las ligeras variaciones, Bull supone que este orden implica tres finalidades esenciales, "ya que su cumplimiento en cierta medida es una condición, no solo para un tipo determinado de vida social, sino para la vida social misma" (p. 56). Estas tres finalidades son, en síntesis: garantizar la vida o la continuación de la existencia frente a la violencia existente o potencial que pueda amenazar la existencia misma; en segundo lugar, está la garantía del cumplimiento de los compromisos adquiridos entre las partes; la tercera finalidad primordial que caracteriza a todo orden social, de acuerdo con Bull, es una especie de combinación entre el respeto a los derechos de propiedad y la importancia del mantenimiento del statu quo. 
Siguiendo este orden de ideas, Bull caracteriza el concepto de orden internacional como "la pauta de actividad acorde con los fines elementales o primarios de la sociedad de Estados, es decir, la sociedad internacional" (p. 60). En este punto es importante mencionar que, de acuerdo con el razonamiento de este autor, los Estados son los principales actores dentro del sistema internacional, y estos se asemejan a individuos dentro de una sociedad determinada, dejando en claro que dentro de las relaciones entre los Estados, a diferencia de los individuos de una sociedad determinada, no existe un poder superior que regule su comportamiento, dos planteamientos que dejan clara la influencia realista en la obra de Bull.

Dejando momentáneamente de lado algunos aspectos conceptuales importantes, es necesario añadir una última categoría de orden, definida por Bull, que denomina el orden mundial, el cual no es más que "los patrones o disposiciones de la actividad humana que cumplen con los que, para la humanidad en su conjunto son los fines elementales o primarios de la vida social” (p. 71).

Esta última categoría de orden hace referencia a un ámbito superior del sistema internacional per se, y es una categoría que se aleja un poco del enfoque grociano y acerca más el concepto kantiano al hacer referencia al concepto de la humanidad, de la "gran sociedad formada por el conjunto de la humanidad" (p. 71). Debido a que no incumbe a la idea principal del presente trabajo, solo se hará una somera mención a esta categoría del orden bulliano.

Una vez hecha esta primera aclaración conceptual sobre el orden, es importante desarrollar el razonamiento seguido por este autor para responder a la segunda pregunta.

En primera instancia, Bull considera que el punto de partida de las relaciones internacionales es la existencia de Estados, considerados como comunidades políticas que gozan de un gobierno propio, autonomía y soberanía sobre un territorio determinado y específico y sobre un segmento de la población que lo habita. Esta diferenciación será fundamental para entender el orden en el siglo XIX, donde por primera vez el mundo experimenta el surgimiento de un sistema político que abarcase al mundo entero. En este punto, es importante hacer la aclaración sobre el concepto de sistema internacional. De acuerdo con Bull,

un sistema de Estados (o sistema internacional) se forma cuando dos o más Estados tienen suficiente contacto entre ellos, y tienen suficiente impacto mutuo sobre las decisiones del otro como para que se comporten -al menos de cierta manera-como partes de un todo (p. 61).

Sin embargo, en el supuesto de un sistema de Estados que excede la sola interacción entre estos, y donde se comparten ciertos intereses, fines y valores comunes, y se gestan ciertas normas comunes que regulan sus relaciones, dicho sistema se convierte en una sociedad internacional. 
Como se mencionó, esta sociedad internacional tiene unos fines primarios para su preservación, que Bull los relaciona de la siguiente manera:

- La conservación del propio sistema y la sociedad de Estados.

- La preservación de la soberanía interna e independencia de cada Estado.

- La preservación de la paz.

Siguiendo esta lógica, Bull considera que desde el siglo XIX han existido dos sociedades internacionales: la sociedad internacional europea, que tuvo lugar en los siglos XVIII y XIX, y la sociedad mundial que tuvo lugar en el siglo XX. ${ }^{3}$

Este orden internacional que estudia Bull goza de una gran estabilidad debido al interés que tienen todos los actores en mantenerlo, aunque no es completamente estático. Bull menciona que dos elementos son fundamentales para el mantenimiento del orden social: instituciones y normas.

A efectos de claridad, este autor define las normas como "principios imperativos generales que exigen o autorizan a determinados tipos de personas o grupos que se comporten de determinada forma" (p. 106) y, claramente, estas normas no solo incluyen las leyes y regulaciones legales, sino también las costumbres, la moral, las buenas formas, los procedimientos o las reglas de juego.

Consecuentemente, la estipulación de dichas normas no significa que los miembros de la sociedad en cuestión vayan a cumplir con ellas, sino que la posibilidad de violar la norma siempre se encuentra latente dentro del comportamiento humano, y en caso de no existir tal posibilidad, argumenta Bull, no tendría sentido la norma en sí. Debido a esta paradójica realidad es que se justifica la existencia de las instituciones como garantes del cumplimiento de dichas normas.

Esto plantea un problema evidente, el cual Bull no ignora, y es que la realidad del concierto internacional es una realidad anárquica y, por ende, carece de cualquier gobierno efectivo que haga valer la efectividad de las normas. Sin embargo, este orden anárquico puede ser explicado, de acuerdo con Bull, por medio de la existencia de fines elementales relacionados con la coexistencia social, que a la larga son los que determinan el comportamiento de todos los miembros de la sociedad. Y en este punto, es importante hacer una anotación: las sociedades anárquicas, así sean las sociedades primitivas o la sociedad internacional, necesitan para su propia subsistencia un reconocimiento de quiénes son sus miembros y, dentro de esos miembros, quiénes son los que pueden desempeñar funciones de poder, siempre según un principio fundamental o constitucional que justifica dicho orden.

${ }^{3}$ Aunque es muy probable que actualmente existan nuevas categorías, considerando que la última vez que Bull propuso las categorías históricas, corría la década de 1980. 
Aunque dentro de las sociedades anárquicas no exista un gobierno central que legisle y ejecute de una manera equitativa para todos los habitantes, las normas dentro de una sociedad anárquica sí existen, en primera instancia, por medio de la moral, de las costumbres y de la censura social. Pero, además, Bull recurre al popular concepto realista de la autoayuda para explicar cómo pueden las normas florecer en un sistema social donde no exista un gobierno, y básicamente se reduce al uso de las medidas consideradas necesarias por una de las partes que sienta que se vulneran sus derechos. En estos casos, el uso de la fuerza se convierte en una opción, debido a la falta de un organismo central que ocupe su monopolio. En el caso particular de la autoayuda, la guerra desempeña un papel predominante. He aquí el aporte liberal a la obra de Bull, incluso es innegable que hay cierta similitud entre su propuesta y el trabajo de Jervis (Jervis, 1978) sobre el juego de la caza del ciervo.

Además, la sociedad internacional tiene una particularidad de la que no goza una sociedad tradicional: sus miembros tienden a ser los mismos perpetuamente. La existencia de todos los miembros de la sociedad internacional difícilmente se alterará, incluso en el futuro lejano. Luego, las relaciones entre todos estos miembros están marcadas por un bagaje importante de repetitivas interacciones históricas y presentes, e incluso futuras considerando la definitiva existencia de estos miembros de la sociedad en el futuro. Desde este punto de vista, entre los Estados del mundo existiría una suerte de “interdependencia”, marcada por ciertas reglas impuestas por la forma como los Estados se relacionan entre sí, dentro del sistema internacional anárquico.

Verbigracia, durante el siglo XIX, el mundo experimentó un cambio de marcada trascendencia. Con el nacimiento de los Estados libres y soberanos comenzó a formarse, con bastantes altibajos y marcados cambios durante el proceso, el sistema internacional moderno. De este modo, el viejo esquema de conquista militar de naciones y otras unidades políticas indeterminadas diferentes de los Estados nación modernos sobre los más débiles era reemplazado por el diálogo entre "actores" reconocidos a lo largo del globo. Esta sería la naciente sociedad internacional actual, donde claramente existirían relaciones de poder, pero las normas que la regirían estarían determinadas por las interacciones entre todos los países. Es importante mencionar en todo caso que estas normas han variado sustancialmente desde aquella época y, lógicamente, el siglo XIX estuvo determinado por los hechos que venían aconteciendo en el siglo pasado y en el antepasado. De esta forma, ya desde hacía doscientos años, se venía gestando un cambio en la forma de relacionarse de los Estados europeos dentro del contexto internacional, principalmente bajo el tratado que fue conocido como la Paz de Westfalia.

Desde el punto de vista de la sociedad internacional, la guerra desempeña un papel significativo, porque su ocurrencia "es una manifestación del desorden en la sociedad internacional y trae consigo la amenaza de que esta entre en crisis” (Bull, 2005, p. 240). 
De hecho, los tres grandes cambios que, a criterio del presente trabajo, experimentó la sociedad internacional a lo largo de los siglos XIX y XX e inicios del siglo XXI, fueron, en su mayoría, signados por guerras: la caída de la sociedad internacional europea se dio como consecuencia de la Primera Guerra Mundial, por un lado; el establecimiento de las grandes organizaciones internacionales que hoy rigen el sistema internacional se dio dentro de la posguerra en la Segunda Guerra Mundial; y la sociedad internacional neoliberal se gestó dentro de la guerra fría.

Del mismo modo, es importante mencionar que la guerra también desempeña un papel disuasivo en la política internacional. Como se ha mencionado, uno de los fines esenciales de la sociedad internacional es su propia supervivencia, lo cual implica la restricción de los actos bélicos en su propia contra.

Pero, claramente, tampoco es posible entender a la sociedad internacional como el "reinado del más fuerte"; las complejidades de las relaciones internacionales entre los distintos miembros que actualmente forman parte de la sociedad internacional impiden que el miembro más fuerte pueda tomar acción con total libertad. La política internacional está signada por un orden, como se ha mencionado, y este orden genera un sistema el cual tiende a ser estable. Esto es posible, porque "el orden en la política internacional depende en primer lugar de determinados hechos contingentes que contribuirían al orden aun así los Estados no percibieran tener intereses, normas e instituciones en común"(Bull, 2005, p. 117).

Esto es claro para el caso de dos Estados fuertes que generen un equilibrio de poder, aunque no se compartan intereses y objetivos comunes en absoluto, y que por ende afecten a todos los demás Estados. Esto se conoce como un hecho contingente y estos hechos contingentes se encuentran presentes a lo largo de todos los grandes sistemas internacionales de la humanidad. La existencia de estos hechos contingentes permite la consolidación de un sistema internacional, pero, en vistas de que no se comparten intereses ni objetivos comunes, no hay lugar para una sociedad internacional. Esta situación podría aplicar para el Imperio romano, donde, a pesar de la supremacía de los romanos, los pueblos que no hacían parte del Imperio no compartían ningún tipo de valor ni interés con los romanos, y, sin embargo, existía un sistema internacional.

Considerando entonces lo que se acaba de exponer, los elementos básicos para componer una sociedad internacional se pueden resumir en tres: la percepción de tener un interés compartido en los fines elementales de la vida social, en las normas que dictan comportamientos acordes con estos fines y en las instituciones que harán las normas efectivas.

Finalmente, Bull reconoce la existencia de las grandes potencias dentro del sistema internacional. Sin embargo, antes de darle la preponderancia que le suelen dar otros autores al estatus de potencia, limita su funcionalidad a un equilibrio de poder y al impacto que tiene dentro del sistema internacional. De esta manera, es posible argumentar que, si bien los Estados Unidos es una superpotencia en el momento, no puede ser el 
dueño del mundo, de lo contrario el sistema internacional no abarcaría dentro de sí a una sociedad internacional, sino a una especie de "feudo" internacional.

\section{Las normas y las instituciones dentro del sistema internacional}

Hedley Bull hace énfasis en la idea de que el comportamiento de los miembros de una sociedad es fundamental para determinar si dicho sistema social alcanza sus fines e intereses compartidos, y también es consciente de que dicho comportamiento no se encuentra naturalmente encadenado al cumplimiento de estos intereses y fines compartidos. Por este motivo es que Bull justifica la existencia de normas dentro de una sociedad que permitan, por lo menos, garantizar al máximo grado que ninguno de los miembros de dicha sociedad verá su existencia en riesgo, que sus derechos de propiedad se respeten y que los pactos que se logren serán cumplidos.

Como se mencionó, dichas normas pueden tener una naturaleza legal, pero no exclusivamente. Vale la pena recordar en este punto que Bull es heredero de la concepción grociana del derecho, según la cual existe un derecho natural que sobrepasa los límites del derecho legal. Siguiendo este orden de ideas, las normas que rigen un sistema social pueden también ser normas de costumbre, normas morales, normas operativas o "reglas de juego", que no necesariamente son elaboradas ante el acuerdo explícito entre las partes ni siquiera como producto de la comunicación entre ellas. Como bien lo menciona Bull, "no es raro que surja una norma, en un primer momento, como norma operativa, se convierta después en una práctica establecida, adquiera más adelante el principio de estatus moral para ser finalmente incorporada en un pacto legal” (p. 119).

Bull hace la siguiente clasificación de normas que han regido el sistema internacional: un primer grupo, que son las que establecen el principio normativo fundamental o constitucional del sistema internacional. Estas se traducen en los principios fundamentales que rigen a la sociedad internacional. Claramente, el reconocimiento de los miembros de dicha sociedad, es decir, los Estados, hace parte fundamental de este tipo de reglas.

En segundo lugar, están las "normas de coexistencia”. Estas normas de coexistencia son las normas aplicables para que los sujetos reconocidos dentro de la sociedad internacional puedan convivir de manera pacífica entre sí. Del mismo modo, el uso de la guerra como última opción, el respeto por los pactos y convenios internacionales y la importancia del territorio y la soberanía para todos los Estados hacen parte de este grupo de normas.

En tercera instancia, están las instituciones, es decir, los órganos encargados de hacer que dichas normas sean cumplidas. En el caso del sistema internacional, Bull establece que en principio estas instituciones son los mismos Estados. De acuerdo con este razonamiento, la generación de normas se da básicamente a través de la costumbre, prácticas establecidas y pactos multilaterales. Es decir que las normas que rigen a la 
sociedad internacional no se encuentran de hecho escritas en ningún código y tampoco existe una institución supranacional que tenga el deber de hacerlas respetar. Es la interacción constante entre los Estados mismos lo que permite que las normas sean respetadas o no. Esto sería fundamental para entender cómo funcionaría la sociedad internacional del siglo XIX y la del siglo XX.

Sin embargo, además de los Estados, existe otro tipo de instituciones, que se vendrían a configurar ante prácticas repetitivas de los Estados. "Por institución no necesariamente entendemos una organización o maquinaria administrativa sino más bien una serie de hábitos y prácticas diseñada para la realización de fines comunes” (Bull, 2005, p. 126). Siguiendo este orden de ideas, Bull relaciona instituciones fundamentales para el sistema internacional, como el equilibrio de poder, el derecho internacional, la diplomacia, la guerra y las grandes potencias. Pero la lista no necesariamente debe detenerse allí, bajo estos parámetros, sino que el comercio y la autoayuda también serían instituciones internacionales, ya que son prácticas que en efecto tienen como objetivo la realización de fines comunes a todos los que la ejercen y, en este trabajo, esta asunción será considerada como válida.

Consecuentemente, cada Estado decide si se adhiere o no a un conjunto de regulaciones y si cumple con dichas regulaciones o no. De la misma manera, la interpretación de aquellas es potestativa de cada Estado y, por ende, la resolución de conflictos se dificulta. Bull expone un ejemplo bastante claro de esta situación cuando comenta que "el principio moral de autodeterminación nacional [...] sustituyó a la legitimidad dinástica, no porque una autoridad legislativa la pusiera en vigor, sino a través de la guerra y la revolución” (p. 125).

Bull, además, trata las organizaciones que son importantes para el sistema internacional tanto desde el punto de vista organizacional, como es el caso de la Organización de las Naciones Unidas, como desde el punto de vista legal, como el derecho internacional, y finalmente trata instituciones más intangibles como el equilibrio de poder o la guerra.

Teniendo en claro lo expuesto hasta el momento, es importante recalcar que Bull, como defensor de la visión grociana de las relaciones internacionales, entiende que la actividad por excelencia de los Estados para relacionarse entre sí no es la guerra como lo afirman los realistas ni tampoco aquella especie de "espíritu universal" que defienden los liberales:

La actividad internacional que, según la visión grociana, mejor ejemplifica la actividad internacional en su conjunto no es ni la guerra entre Estados, ni el conflicto horizontal que atraviesa la frontera entre los Estados, sino el comercio, o de forma más general, el intercambio económico y social (p. 78).

Sin embargo, a pesar de darle reconocimiento al comercio como la actividad central de la interacción entre los Estados dentro de la visión grociana de las relaciones 
internacionales, Bull no hace mayor mención a este dentro de su análisis sobre el orden internacional, y se centra en otros aspectos, los cuales, si bien son fundamentales para el orden internacional, no tienen relación con la forma como los Estados principalmente interactúan entre sí: el comercio. Los seguidores de la Escuela Inglesa de Relaciones Internacionales han denominado racionalista esta tendencia grociana (Knudsen, 2000).

A pesar de su temprana muerte, el legado de Bull ha sido determinante para la llamada Escuela Inglesa de las Relaciones Internacionales, pero su uso de fuentes tan diversas como Grocio (1625) y Oppenheim (1905), y de pensar en una opción diferente, un término medio si se quiere, de las tendencias que Wight denominó el realismo y el revolucionismo (liberalismo), ha llevado a sus seguidores a tomar posiciones muy distintas sobre la Escuela Inglesa de las Relaciones Internacionales.

Muchos de sus seguidores, como Buzan (2001) o Jackson (2000), han mostrado fuerte influencia de autores realistas, como Hoffman (1986), Morgenthau (1978) y Waltz (1979), del mismo modo como Dunne (1998) ha tomado influencias de Wendt (1992), mientras que la denominada concepción "solidarista" de la Escuela Inglesa de las Relaciones Internacionales, término acuñado por el mismo Bull (Knudsen, 2000), es más cercana a los liberales.

\section{Conclusiones}

La propuesta de Bull, si bien es cierto que supera ciertas falencias de realismo y liberalismo, no está exenta de críticas y debilidades. Para empezar, se podría señalar la más obvia: la falta de estudios. Keohane y Martin (1995) admitían que una de las grandes debilidades de su teoría de la interdependencia compleja es que aún no se habían llevado a cabo estudios suficientes que pudieran dar mayor fortaleza a su argumento y que aún era necesario "integrar consideraciones distribucionales en sus modelos, especificar con mayor profundidad los mecanismos causales por medio de los cuales las instituciones ejercen influencia y construir sobre trabajo empírico existente para mostrar evidencia más contundente sobre los efectos institucionales" (p. 51). Esta falencia es aún mayor en el trabajo de Bull. Su repentina muerte en 1985 privó a la comunidad académica de trabajos adicionales donde pudiera ofrecer mayores especificaciones y aclaraciones sobre muchos de sus planteamientos, así como dificultó la elaboración de estudios adicionales que pudieran demostrar empíricamente sus formulaciones teóricas. Incluso su amigo y colega el realista Stanley Hoffman admitió que probablemente esta era la mayor debilidad del académico australiano: "Siempre fui un gran admirador de la extraordinaria agudeza de la mente de Bull, sin embargo, cuando reviso su trabajo tengo una sensación inevitable de inconclusión” (1986, p. 179).

Aunado a lo anterior, conceptualmente Bull deja muchos interrogantes en el aire. Por ejemplo, si se consideraba un seguidor de la visión grociana de las relaciones internacionales, la cual pone un gran énfasis en el intercambio comercial, ¿̇por qué no trató 
la institución del comercio en su libro La sociedad anárquica? ¿No debería ser esta la institución más importante dentro del sistema internacional? ¿Qué criterios se deben utilizar para determinar cuándo un comportamiento se convierte en norma y cuándo una norma se convierte en institución? ¿Se puede perder la calidad de institución? Considerando que en la introducción del libro Bull menciona que el orden es solo un aspecto de la política internacional, ¿qué otros aspectos no tratados por Bull abarca la política internacional y cuál es la relación entre estos? Son solo algunos de los interrogantes que la propuesta de Bull deja en el aire.

Sin embargo, a pesar de sus grandes debilidades, la propuesta bulliana no debería ser ignorada. La existencia de una realidad internacional guiada por normas e instituciones más que por los designios de las grandes potencias o por fórmulas mágicas de desarrollo y paz puede ser una alternativa para entenderla más a fondo.

Ya a un nivel más práctico es posible entender cómo han funcionado las normas e instituciones dentro de estos dos siglos de existencia del sistema internacional mundial. Por ejemplo, el Tratado Interamericano de Asistencia Recíproca, más que una jugada de los Estados Unidos para salvaguardar sus intereses en su patio trasero (la cual es la explicación realista, vista desde la óptica latinoamericana), podría considerarse como un resultado de las normas sobre seguridad colectiva de la época de la guerra fría, las cuales son muy distintas del momento en que los Estados Unidos llama a la coalición contra el terrorismo en la era pos-11/9.

Mientras que las normas del sistema internacional de la guerra fría requerían respuestas militares internacionales conjuntas contra la invasión de un enemigo y una ideología claramente identificables en un país o países determinados y un uso mayor de movidas estratégicas, las normas del sistema internacional en la guerra contra el terrorismo implican un mayor énfasis en lo táctico, para luchar contra un enemigo que no es claramente identificable con un país particular y donde un compromiso en bloque no produciría resultados contundentes, ya que el terrorismo, en teoría, puede atacar en cualquier parte y no tiene necesariamente el objetivo de obtener el dominio político de un territorio particular por la fuerza.

Del mismo modo, la Alianza para el Progreso, que se formuló a mediados del siglo pasado, puede no haber funcionado en Latinoamérica debido al mal comportamiento de la región (explicación de cuño liberal), sino que probablemente esta iniciativa estaba circunscrita bajo una serie de normas internacionales que son muy distintas de las normas que regían al mundo en ese entonces, las cuales, aunque propuestas por los mismos organismos, diferían mucho de las normas imperantes cuando se propuso la serie de medidas que harían parte del famoso Consenso de Washington, casi cuarenta años después.

Para la década de 1950, el comercio mundial seguía registrando niveles bajos, ya habían accedido nuevos competidores (los países africanos y asiáticos recién 
independizados) a los mercados internacionales de productos agrícolas y minerales y las políticas de restricción de importaciones y redistribución de la riqueza propias del estado de bienestar todavía tenían mucha fuerza. Europa ya estaba dando pasos en dirección a la Política Agrícola Comunitaria, a la vez que los esfuerzos por crear una institución internacional que rigiera el comercio entre los Estados no obtenía el apoyo suficiente. Por otro lado, los Estados Unidos subsidiaban a sus productores, quienes tenían mayores ventajas en su propio mercado, y estaban impulsando con éxito el Plan Marshall en Europa. Considerando que el modelo de industrialización dirigida por el Estado (Ocampo, 2004) restringía aún más los niveles de comercio internacional en Latinoamérica, y que solo las tres economías principales tímidamente implementaron un modelo de fortalecimiento de la industria, pero que al final demostró ser insostenible, es posible afirmar que la cantidad de dinero aportada por los Estados Unidos para el desarrollo de la región habría sido irrelevante, ya que con estos aportes o sin ellos no habría sido posible alcanzar desarrollo en esas condiciones.

Del mismo modo, en el caso del Consenso de Washington, donde ya primaban las normas internacionales de estilo neoliberal, el comercio internacional estaba mucho más liberado, el sistema financiero estaba recibiendo un impulso fulminante y los precios de los productos agrícolas caían con fuerza, mientras los precios de los productos minerales no eran estables, por lo que la solución más liberal que se encontró no trataba tanto de un mayor apoyo económico de las grandes potencias, sino de medidas para que los países en crisis pudiesen superar solos sus adversidades. Por ende, el fomento de las inversiones de cartera, la fijación de tasas de cambio competitivas, la responsabilidad fiscal y la reducción del paquidérmico Estado de mediados del siglo XX fueron propuestas con el ánimo de lograr que Latinoamérica obtuviese capital económico de manera pronta. Actualmente, en un mundo donde rigen normas internacionales que difieren de hace veinte o cincuenta años, donde el comercio internacional está aún más liberado, pero hay mayor regulación del mercado financiero y donde el mayor flujo de capital viene de manos de la empresa privada y no del Estado, propuestas como las del expresidente argentino Nestor Kirschner de implementar un segundo Plan Marshall en América Latina a inicios del siglo XXI no habría traído los resultados esperados.

Como se puede ver, es posible hacer una interpretación plausible de la realidad latinoamericana desde la óptica de las normas y de las instituciones bullianas, que se aleje de los tradicionales enfoques realista y liberal, de momento no resta sino esperar que se adelanten estudios correspondientes que permitan darle un apropiado impulso a esta propuesta.

\section{Referencias}

Arias, Ó. (2009). “Algo hicimos mal”. En nacion.com. Recuperado de http://wvw.nacion. com/ln_ee/2009/abril/26/opinion1944940.html 
Axelrod, Robert (2006). The evolution of cooperation. Basic Books.

Bull, H. (2005). La sociedad anárquica: un estudio sobre el orden en la política mundial. Madrid: Catarata.

Buzan, B. (1983). People, State and Fear. Brighton, Sussex: Wheatsheaf Books.

Buzan, B. (2001). The English School: an underexploited resource in IR. Review of International Studies, 27(03), 471-488.

Carr, E. H., (1964). The twenty years' crisis, 1919-1939: an introduction to the study of international relations. Nueva York: Harper \& Ro.

Calderón, H. (2009). La situacion en México y el impacto del narcoterrorismo en el continente americano. Recuperado de http://foroazulyblanco.blogspot.com. co/2009/o4/narcoterrorismo-en-el-continente.html

Chávez Frías, H. (2013). Médicos para la humanidad. En Revolución o Muerte. Recuperado de http://www.revolucionomuerte.org/index.php/discursos/ discursos-comandante-hugo-chavez/1612-medicos-para-la-humanidad-comandante-hugo-chavez-frias-acto-de-graduacion-de-medicos-integrales-comunitarios

Doyle, M. W. (1986). Liberalism and world politics. American Political Science Review, $80(4), 1151-1169$.

Dunne, T. (1998). Inventing international society: A history of the english school. Londres: St Antony's College.

Escudé, C. (1998). An introduction to peripheral realism and its implications for the interstate system: Argentina and the Condor II Missile Project. En S. Neuman (ed.), International relations theory and the Third World (pp. 55-75). Nueva York: St. Martin's Press.

Galeano, E. (2004). Las venas abiertas de América Latina. México, Buenos Aires: Siglo XXI.

Gilpin, R. (1983). War and change in world politics. Cambridge: Cambridge University Press.

Grieco, J. M. (1988). Anarchy and the limits of cooperation: A realist critique of the newest liberal institutionalism. International Organization, 42(03), 485-507.

Grocio, H. (1925). Del derecho de la guerra y de la paz. Ontario: Batoche Books.

Haas, E. B. (1958). The Uniting of Europe: Political, economic and social forces, 19501957. Londres: Stevens \& Sons.

Hoffman, S. (1986). Hedley Bull and his contribution to international relations. International Affairs (Royal Institute of International Affairs 1944-), 62(2), 179-195. 
Jackson, R. (2000). The global covenant: Human conduct in a world of states. Oxford: Oxford University Press.

Jenkins, B. M. (2014). Disrupting terrorist safe havens. Recuperado de http://www. rand.org/blog/2014/o8/disrupting-terrorist-safe-havens.html

Jervis, R. (1978). Cooperation under the security dilemma. World Politics, 3O(02), 167-214.

Keohane, R. O. (1984). After hegemony: Cooperation and discord in the world political economy. Princeton, N.J.: Princeton University Press.

Keohane, R. O. y Martin, L. L. (1995). The promise of institutionalist theory. International Security, 2o(1), 39-51.

Keohane, R. O. y Nye, J. (1977). Power and independence: World politics in transition. Boston: Little, Brown.

Krasner, S. D. (1983). International regimes. Ithaca y Londres: Cornell University Press.

Knudsen, T. B. (2000). International Society and International Solidarity: Recapturing the Solidarist Origins of the English School. Ponencia presentada en 28th Joint Sessions of Workshops of the European Consortium for Political Research, Copenhagen, Dinamarca.

Mearsheimer, J. J. (1994). The false promise of international institutions. International Security, 19(3), 5-49.

Mearsheimer, J. J. (2001). The tragedy of great power politics. Nueva York: WW Norton \& Company.

Mittrany, D. (1994). A working peace system. En B. F. Nelsen y A. C-G. Stubb, The European Union (pp. 77-97). Londres: Macmillan Education UK.

Moravcsik, A. (1992). Liberalism and international relations theory. Cambridge, MA: Center for International Affairs, Harvard University.

Morgenthau, H. (1978). Politics amongst nations: the struggle for power and peace. Alfred A. Knopf

Mujica, J. (25 septiembre 2013). Discurso ante la ONU. Recuperado de http://www. lr21.com.uy/politica/1131614-mujica-en-la-onu-discurso-completo

Nye, J. S. (1969). United States policy toward regional organization. International Organization, 23(03), 719-740.

Ocampo, J. A. (2004). La América Latina y la economía mundial en el largo siglo XX. El Trimestre Económico, 71, 284(4), 725-786. 
Oppenheim, L. (1920). International law: A treatise. Longmans, Green and Company.

Oye, K. A. (1985). Explaining cooperation under anarchy: Hypotheses and strategies. World Politics, 38(1), 1-24.

Ricardo, D. (2001). Principles of political economy and taxation. Ontario: Batoche Books.

Thompson, K. y Morgenthau, H. (1985). Politics among nations: The struggle for power and peace. Alfred A. Knopf.

Waltz, K. (1979). Theory of international politics. Nueva York: McGraw Hill.

Wendt, A. (1992). Anarchy is what states make of it: The social construction of power politics. International Organization, 46(02), 391-425.

Wilson, W. (8 enero 1918). President's Woodrow Wilson 14 points. Recuperado de http://avalon.law.yale.edu/2oth_century/wilson14.asp

Wohlforth, W. C. (1999). The stability of a unipolar world. International Security, 24(1), 5-41. 